\title{
Biodegradable mesoporous calcium-magnesium silicate-polybutylene succinate scaffolds for osseous tissue engineering
}

This article was published in the following Dove Press journal:

International Journal of Nanomedicine

28 October 2015

Number of times this article has been viewed

\author{
Xinxin Zhang ${ }^{1,2, *}$ \\ Chi Zhang,* \\ Wei Xul,* \\ Biao Zhong ${ }^{3}$ \\ Feng $\operatorname{Lin}^{3}$ \\ Jian Zhang ${ }^{3}$ \\ Quanxiang Wang ${ }^{4}$ \\ Jiajin $\mathrm{Ji}^{4}$ \\ Jie $\mathrm{Wei}^{4}$ \\ Yang Zhang' \\ 'TongRen Hospital, School of \\ Medicine, Shanghai Jiao Tong \\ University, ${ }^{2}$ Shanghai Institute of \\ Materia Medica, Chinese Academy \\ of Sciences, ${ }^{3}$ Shanghai Sixth \\ People's Hospital, Shanghai Jiao \\ Tong University, ${ }^{4}$ Key Laboratory \\ for Ultrafine Materials of Ministry \\ of Education, East China University \\ of Science and Technology, Shanghai, \\ People's Republic of China \\ *These authors contributed equally \\ to this work
}

Correspondence: Yang Zhang

Tongren Hospital, School of Medicine,

Shanghai Jiao Tong University,

IIII Xianxia Road, Shanghai 200336,

People's Republic of China

Tel +86 2I 52039999

$\mathrm{Fax}+862160906478$

Email zy_benjamin@।63.com

Jie Wei

Key Laboratory for Ultrafine Materials of Ministry of Education, East China

University of Science and Technology,

Shanghai 200237, People's Republic of

China

Tel +86 2I $6425 \quad 308$

Fax +86 2I 6425 I358

Email jiewei7860@sina.com

\begin{abstract}
The structural features of bone engineering scaffolds are expected to exhibit osteoinductive behavior and promote cell adhesion, proliferation, and differentiation. In the present study, we employed synthesized ordered mesoporous calcium-magnesium silicate (om-CMS) and polybutylene succinate (PBSu) to develop a novel scaffold with potential applications in osseous tissue engineering. The characteristics, in vitro bioactivity of om-CMS/PBSu scaffold, as well as the cellular responses of MC3T3-E1 cells to the composite were investigated. Our results showed that the om-CMS/PBSu scaffold possesses a large surface area and highly ordered channel pores, resulting in improved degradation and biocompatibility compared to the PBSu scaffold. Moreover, the om-CMS/PBSu scaffold exhibited significantly higher bioactivity and induced apatite formation on its surface after immersion in the simulated body fluid. In addition, the om-CMS/PBSu scaffold provided a high surface area for cell attachment and released $\mathrm{Ca}$, $\mathrm{Mg}$, and $\mathrm{Si}$ ions to stimulate osteoblast proliferation. The unique surface characteristics and higher biological efficacy of the om-CMS/PBSu scaffold suggest that it has great potential for being developed into a system that can be employed in osseous tissue engineering.
\end{abstract}

Keywords: bone repair, polybutylene succinate, calcium-magnesium silicate, ordered mesoporous, proliferation

\section{Introduction}

The treatment of bone defects resulting from trauma, infections, tumors, or genetic malformations represents a major challenge for clinicians. A three-dimensional structure known as a "scaffold", fabricated from a suitable artificial or natural material and exhibiting high porosity and pore interconnectivity, has been widely studied to regenerate the lost bone and provide treatment modalities for bone defects. ${ }^{1,2}$ Scaffolds should ideally not only provide a passive structural support for bone cells but they should also favorably modulate bone formation by simulating osteoblastic cell proliferation and differentiation. ${ }^{3}$ Scaffolds have certain advantages over autologous grafts, such as easy shaping, limitless supply, and avoidance of donor-site morbidity. Biodegradable scaffolds even allow new tissues to take over the biological functions; therefore, potential chronic problems associated with biostable implants can be avoided. ${ }^{4,5}$

Multiple attempts have been made to construct scaffolds exhibiting the desired porosity and mechanical performance by using inorganic bioactive ceramics and glass or from biodegradable polymers. ${ }^{6,7}$ Polybutylene succinate (PBSu) is a biodegradable biopolymer with good processability, excellent mechanical properties, and harmless degradation products $\left(\mathrm{CO}_{2}\right.$ and $\left.\mathrm{H}_{2} \mathrm{O}\right) .{ }^{8} \mathrm{PBSu}$ has been studied extensively for its potential to be used as a conventional plastic and is also proposed to be a good candidate 
for use as a supporting material that has various applications in the field of tissue engineering. Notably, Wang et al have reported the use of $\mathrm{PBSu}$ based scaffolds in artificial bone implants. ${ }^{9}$ Although PBSu is an excellent material for use as a bone substitute and possesses certain controllable manufacturing characteristics on the large scale, its osteocompatibility is still insufficient. Some clinical studies have reported that the degradation rate of $\mathrm{PBSu}$ polymer material varies greatly between patients, and the void left after polymer degradation was not filled by regenerating bone. ${ }^{10-12}$

Currently, the research on the use of bioglass for biomedical purposes has largely focused on new compositional modifications, processing, and potential biomedical applications. Silicate glasses that are based primarily on the $\mathrm{SiO}_{2}-\mathrm{CaO}-\mathrm{Na}_{2} \mathrm{O}-\mathrm{P}_{2} \mathrm{O}_{5}$ system exhibit unique biological properties, such as osteoinductive behavior and the ability to bind to soft tissue as well as to hard tissue when exposed to biological fluids. ${ }^{13}$ The ionic dissolution products from bioglass (eg, Si, Ca, P) stimulate the expression of osteolastic cells and angiogenesis, leading to the formation of strong bonds between the bioactive glass and human bone. ${ }^{14}$ Further, many trace elements present in the human body such as $\mathrm{Mg}, \mathrm{Cu}, \mathrm{Zn}$, and $\mathrm{Sr}$ are known for their anabolic effects on bone metabolism. ${ }^{15}$ Therefore, novel techniques that enhance bioactivity of scaffold materials by introducing therapeutic ions are now being investigated. The release of these ions upon exposure to a physiological environment is believed to favorably affect the behavior of human cells and also enhance the bioactivity of these scaffolds in stimulating both osteogenesis and angiogenesis.

Herein, we report the development of a novel scaffold comprising a combination of active ordered mesoporous calcium-magnesium silicate (om-CMS) material with $\mathrm{PBSu}$. This om-CMS/PBSu scaffold was expected to exhibit sustained release of the mesoporous cargo from the polymeric scaffold and stimulate cell adhesion, proliferation, and differentiation into osteoblasts. We have also evaluated the characteristics of the scaffold material, its mechanical properties, and cellular responses.

\section{Materials and methods Preparation of om-CMS/PBSu scaffold}

om-CMS was synthesized using the typical sol-gel method. ${ }^{16}$ Briefly, $4 \mathrm{~g}$ of Pluronic P123 $\left(\mathrm{PEO}_{20}-\mathrm{PPO}_{70}-\mathrm{PEO}_{20}\right.$, SigmaAldrich Co., St Louis, MO, USA) was dissolved in $30 \mathrm{~g}$ of $\mathrm{H}_{2} \mathrm{O}$ and $120 \mathrm{~g}$ of $\mathrm{HCl}$ solution $(2 \mathrm{M})$, and mixed well. This solution was then stirred until clear and then $4.8 \mathrm{~g}$ of $\mathrm{MgNO}_{3} \cdot 6 \mathrm{H}_{2} \mathrm{O}$ and $5.2 \mathrm{~g}$ of $\mathrm{CaNO}_{3}$ were added to the solution. This was followed by addition of $8.5 \mathrm{~g}$ of tetraethyl orthosilicate dropwise using an injection pump. The reaction mixture was vigorously stirred at $50^{\circ} \mathrm{C}$ for 5 hours and then incubated for another 24 hours at $80^{\circ} \mathrm{C}$ to obtain precipitation. Finally, the precipitate was filtered out, washed thoroughly with deionized water, and dried in vacuum at $60^{\circ} \mathrm{C}$ to obtain the om-CMS powder.

The om-CMS/PBSu scaffold was prepared by mixing the om-CMS and PBSu powders in a ratio of 40:60 by weight at $130^{\circ} \mathrm{C}$ for 10 minutes. Disc-shaped scaffold samples $(\Phi 15 \times 2 \mathrm{~mm})$ were then prepared at $130^{\circ} \mathrm{C}$ using a mold. The samples were then washed with ultrasounds for 10 minutes and dried in an oven at $37^{\circ} \mathrm{C}$ to obtain the composites.

\section{Characterization}

The morphology and microstructure of om-CMS and om-CMS/PBSu were observed by transmission electron microscopy. The transmission electron microscopy micrographs were obtained on a JEM1400F electron microscope (JEOL, Tokyo, Japan) with an accelerating voltage of $200 \mathrm{kV}$. The porosities and specific surface area of the omCMS were measured through nitrogen adsorption-desorption isotherms by using a Macromeritics ASAP2010N analyzer. The surface areas of the powders were calculated by the Brunauer-Emmett-Teller equation. ${ }^{17}$ The relative pressure $\mathrm{P} / \mathrm{Po}$ of the isotherms was studied between 0.01 and 1.0 . The pore parameters were calculated by employing the BarrettJoyner-Halenda model. ${ }^{18}$ The chemical structure and functional groups of the om-CMS, PBSu, and om-CMS/PBSu composites were characterized by Fourier transform infrared spectroscopy (FTIR, Thermo Nicolet 6700, Waltham, MA, USA). The phase and crystallographic structures of om-CMS, $\mathrm{PBSu}$, and om-CMS/PBSu were characterized by X-ray diffraction using the $\mathrm{D} / \mathrm{max} 2550 \mathrm{VB} / \mathrm{PC}$ diffractometer (Rigaku, Tokyo, Japan) with a scan range from $10^{\circ}$ to $80^{\circ}$.

\section{In vitro biodegradation}

An immersion test was performed to evaluate degradation, bioactivity, and mechanical stability of the om-CMS/PBSu scaffold by immersing the samples in simulated body fluid (SBF). The standard SBF solution was prepared by dissolving $\mathrm{NaCl}, \mathrm{NaHCO}_{3}, \mathrm{KCl}, \mathrm{K}_{2} \mathrm{HPO}_{4} \cdot 3 \mathrm{H}_{2} \mathrm{O}, \mathrm{MgCl}_{2} \cdot 6 \mathrm{H}_{2} \mathrm{O}$, $\mathrm{CaCl}_{2} \cdot 6 \mathrm{H}_{2} \mathrm{O}$, and $\mathrm{Na}_{2} \mathrm{SO}_{4}$ in distilled water and buffering it to a pH of 7.4 with Tris buffer and $1 \mathrm{~N} \mathrm{HCl}$ as described previously by Kokubo and Takadama. ${ }^{19}$ The ionic concentration of SBF simulates that of human plasma. Circular tablets of om-CMS/PBSu were immersed in flasks containing SBF at $37^{\circ} \mathrm{C}$. The morphologies of the scaffolds before and after the 
SBF immersion test were observed under a scanning electron microscope (VEGA TS 5163MM, TESCAN, Brno, Czech Republic) at an accelerating voltage of $20 \mathrm{kV}$. A pH meter was used to monitor changes of $\mathrm{pH}$ in the solution. The amount of weight lost by the samples during the experiment was calculated as the difference in weight of samples before and after immersion in the SBF.

\section{Structural and element stability}

The phase structure of the om-CMS/PBSu scaffold after the SBF immersion test was evaluated by using X-ray diffraction. To evaluate the exchange of ions between the samples and the solutions, the chemical composition of the soaking solutions was analyzed by using an inductively coupled plasma spectrometer (JY70 Plus, Horiba Jobin Yvon, Palaiseau, France) to measure the amount of $\mathrm{Ca}, \mathrm{Mg}$, and $\mathrm{Si}$ in the soaking solutions.

\section{Cell culture}

The mouse osteoblastic cell line MC3T3-E1 was used to investigate the interaction between osteoblasts and the om-CMS/PBSu composite. The cells were cultured in modified Eagle's medium supplemented with $10 \%$ fetal bovine serum, $100 \mu \mathrm{g} / \mathrm{mL}$ penicillin, and streptomycin at $37^{\circ} \mathrm{C}$ in an environment of $5 \% \mathrm{CO}_{2}$ and saturated humidity. The culture medium was replaced with fresh medium every 3 days.

\section{Cell adhesion and imaging}

To study the influence of serum proteins fibronectin (FN) and scaffold on the osteoblast cell attachment and proliferation, we have processed the om-CMS/PBSu composite using a simple treatment. The om-CMS/PBSu samples were placed in a 24-well plate and sterilized in 75\% ethanol for 1 hour and then washed twice with phosphate buffered saline liquid to remove residual ethanol. The substrates were then incubated with either $10 \%$ FBS or serum levels of FN $(20 \mu \mathrm{g} / \mathrm{mL})$ for 4 hours at $37^{\circ} \mathrm{C}$. After this time period, each substrate was gently washed with phosphate buffered saline just before seeding with cells.

The MC3T3-E1 cells were seeded on these substrates at a density of $10^{4}$ cells $/ \mathrm{cm}^{2}$ and incubated for 6 hours. The substrates were then washed twice with serum-free medium. Cells were fixed with $4 \%$ paraformaldehyde for 10 minutes, then washed three times with phosphate buffered saline, and permeabilized with $0.1 \%$ Triton X-100 for 10 minutes. Cellseeded samples were stained with $20 \mu \mathrm{g} / \mathrm{mL}$ of Acti-stain 488 phalloidin (Sigma-Aldrich) and then counterstained with $1 \mu \mathrm{g} / \mathrm{mL}$ of DAPI for 5 minutes at room temperature.
The cells were imaged by using a confocal laser-scanning microscope (Leica Microsystems, Wetzlar, Germany).

\section{Cell proliferation}

Cells were cultured on the PBSu and om-CMS/PBSu substrates at a density of $10^{4}$ cells $/ \mathrm{cm}^{2}$ and cell proliferation was measured at 1, 4, and 7 days using the Cell Counting Kit-8. ${ }^{20}$ After culturing on substrates, the Cell Counting Kit-8 solution $(5 \mathrm{mg} / \mathrm{mL})$ was added to each well $(100 \mu \mathrm{L} /$ well $)$, and the cells were incubated for another 4 hours. The optical density (OD) of each well at $450 \mathrm{~nm}$ was determined using a spectrophotometer (BioTek, Winooski, VT, USA).

\section{Statistical analysis}

The data were analyzed by a one-way analysis of variance using SPSS 10.0 (SPSS Inc., Chicago, IL, USA). The results of the statistical analysis are expressed as mean \pm standard deviation. Differences were considered statistically significant at a $P$-value $<0.05$.

\section{Results and discussion Characterization of om-CMS/PBSu}

Figure 1A shows the images of PBSu and om-CMS/PBSu scaffolds. The density of the PBSu scaffold increases from 1.26 to $1.42 \mathrm{~g} / \mathrm{cm}^{3}$ after blending with the om-CMS powder. Figure 1B and $\mathrm{C}$ represents transmission electron microscopy images revealing the morphology and microstructure of the om-CMS/PBSu scaffold. An ordered mesoporous structure with a uniform pore size can be clearly observed. $\mathrm{N}_{2}$ adsorption-desorption isotherms of the om-CMS/PBSu scaffold (Figure 2A) revealed that the om-CMS/PBSu scaffold exhibits a type IV adsorption-desorption isotherm with H1-type hysteresis loops at high relative pressures according to International Union of Pure and Applied Chemistry classification. This would indicate the presence of independent cylindrical slim pore channels with a highly uniform pore size distribution. ${ }^{21}$ Both branches of the adsorption-desorption isotherms showed a sharp adsorption-desorption step in the $\mathrm{P} / \mathrm{P} 0$ region between 0.8 and 1.0. These results suggest that the om-CMS/PBSu scaffold possesses a well-defined array of regular mesopores. The pore size distribution calculated from the adsorption-desorption isotherm by the BarrettJoyner-Halenda method is shown in Figure 2B. It was found that the om-CMS/PBSu scaffold exhibits a narrow pore size distribution with an average pore size of approximately $13 \mathrm{~nm}$ and a relatively large Brunauer-Emmett-Teller surface area of $891 \mathrm{~m}^{2} / \mathrm{g}$. This ordered mesoporous structure and large specific surface area of the scaffold are conducive for the 


\section{A}

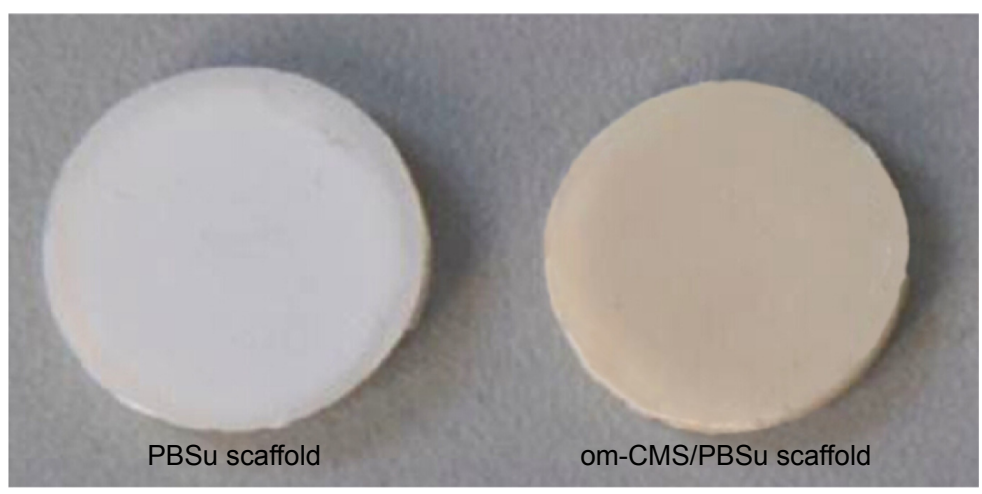

B

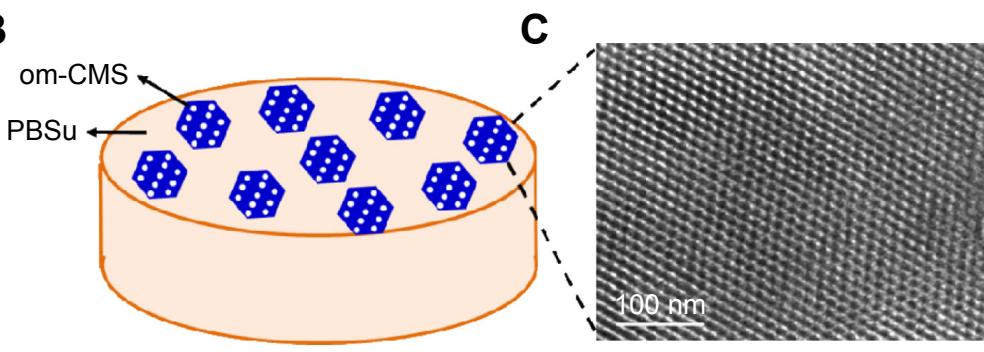

Figure I (A) Photographs of PBSu scaffold and om-CMS/PBSu scaffold. (B) Schematic illustration of om-CMS/PBSu composite. (C) Ordered mesoporous with uniform pore size can be clearly observed from (C) TEM images.

Abbreviations: PBSu, polybutylene succinate; om-CMS, ordered mesoporous calcium-magnesium silicate; TEM, transmission electron microscopy.

transmission of body fluids to the site of bone defect, and thus promote cell adhesion and proliferation. ${ }^{22}$ Furthermore, the large specific surface area of the scaffold enhances the probability of contact with body fluids ions, which accelerate the degradation of the scaffold material. This results in excellent biological activity and osteocompatibility.

FTIR spectra of PBSu, om-CMS, and om-CMS/PBSu are shown in Figure 3. The IR bands at 1,717 and $1,148 \mathrm{~cm}^{-1}$ represent the vibrational mode of the ester base of $\mathrm{PBSu}$ (Figure $3 \mathrm{~A}$ ). The stretching vibration peak of the $\mathrm{Si}-\mathrm{O}$ structure in om-CMS was seen at $1,047 \mathrm{~cm}^{-1}$ (Figure 3B).
All these peaks were observed in the FTIR spectrum of om-CMS/PBSu (Figure 3C), suggesting that the effects of PBSu and om-CMS get compounded when mixed together. Figure 4 shows the X-ray diffraction pattern of PBSu, om$\mathrm{CMS}$, and om-CMS/PBSu. It was observed that om-CMS had a broad peak at approximately $22^{\circ}$, which is typical for amorphous silicate materials. ${ }^{23}$ Strong diffraction peaks at $19^{\circ}$ and $22^{\circ}$ were observed for PBSu. Notably, the diffraction peaks obtained from om-CMS/PBSu were broader and less intense than the sample PBSu, indicating that om-CMS/ $\mathrm{PBSu}$ is less crystalline than PBSu.
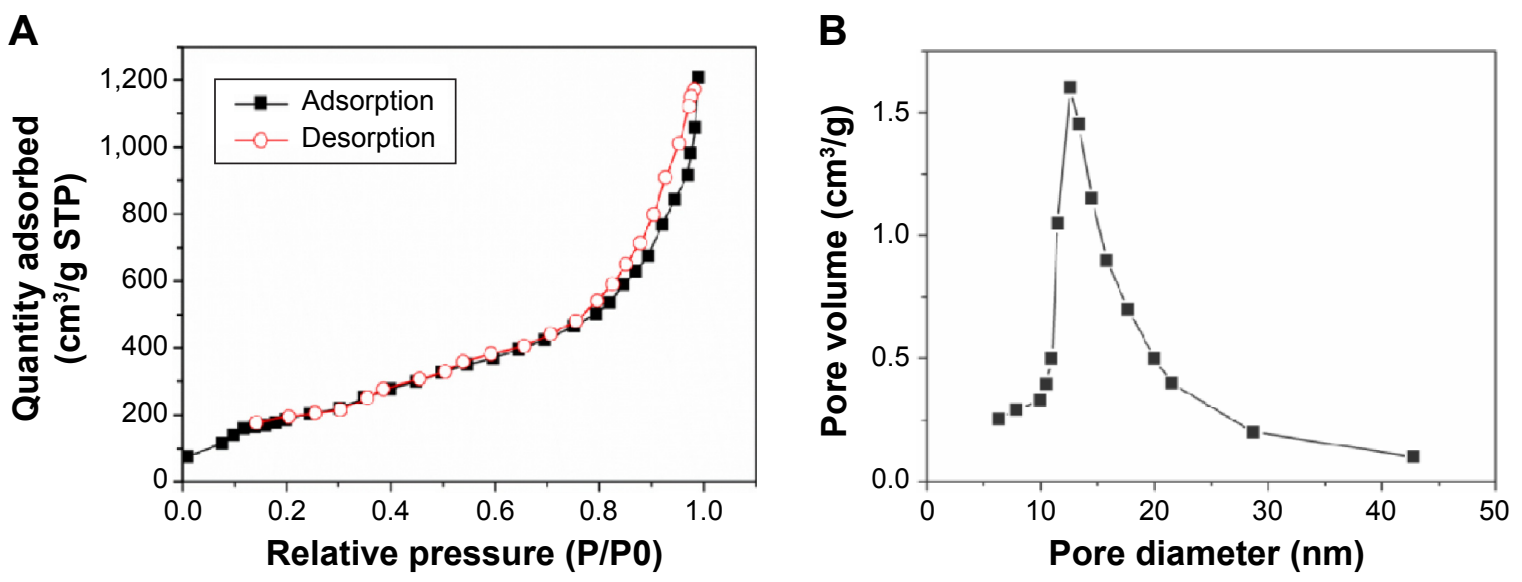

Figure 2 N2 adsorption-desorption isotherms (A) and pore size distribution (B) of the om-CMS/PBSu scaffold. Abbreviations: om-CMS, ordered mesoporous calcium-magnesium silicate; PBSu, polybutylene succinate. 

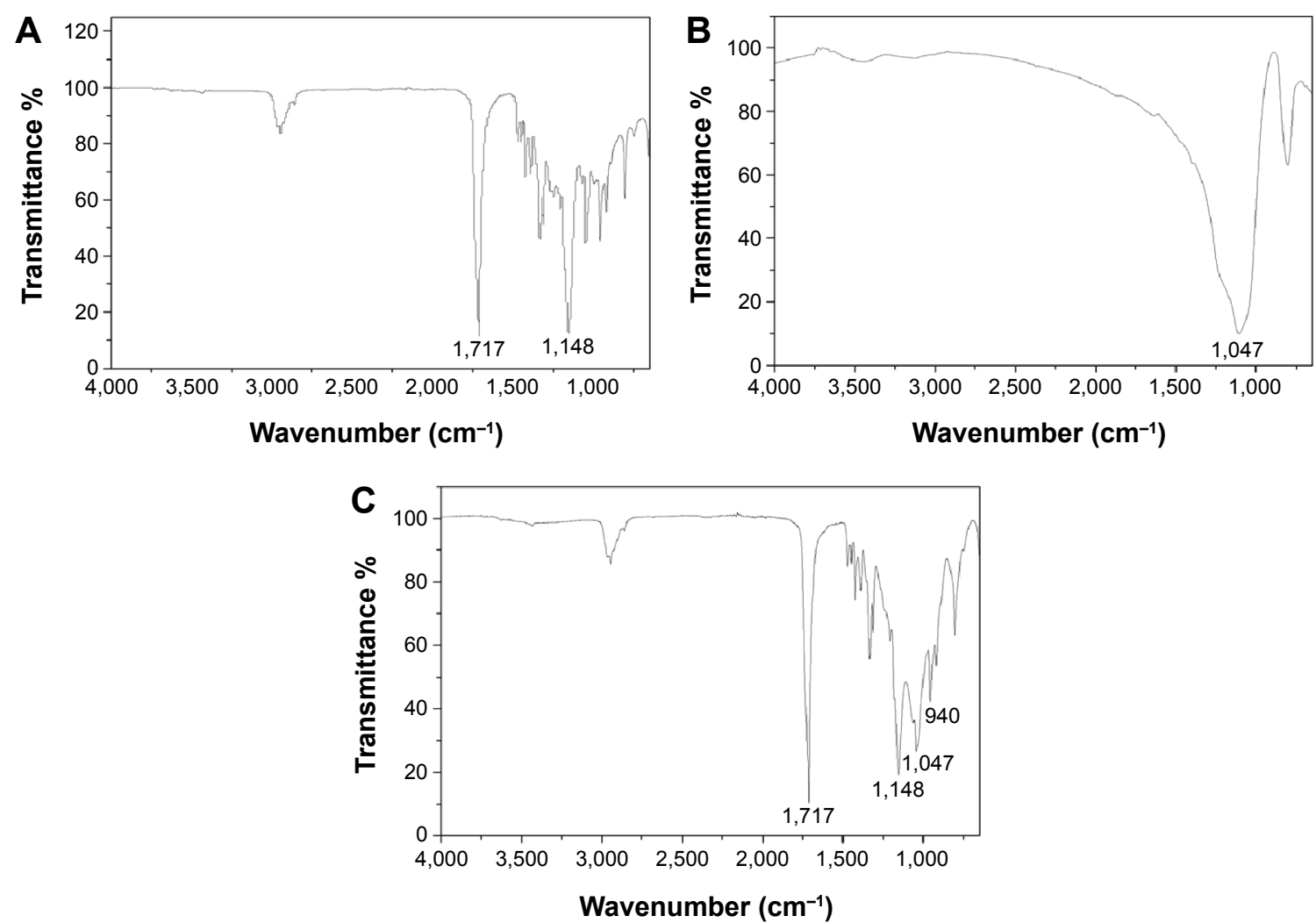

Figure 3 FTIR spectra of om-CMS (A), PBSu (B), and om-CMS/PBSu (C).

Abbreviations: FTIR, Fourier transform infrared spectroscopy; om-CMS, ordered mesoporous calcium-magnesium silicate; PBSu, polybutylene succinate.

\section{Degradability of om-CMS/PBSu in the SBF} Degradability of a biomaterial in a physiological environment is crucial for its application in tissue engineering. Immersion tests in SBF are the standard procedure to estimate the in vitro biodegradability and bioactivity of scaffolds. This provides information on the long-term degradation characteristics of the implants, including

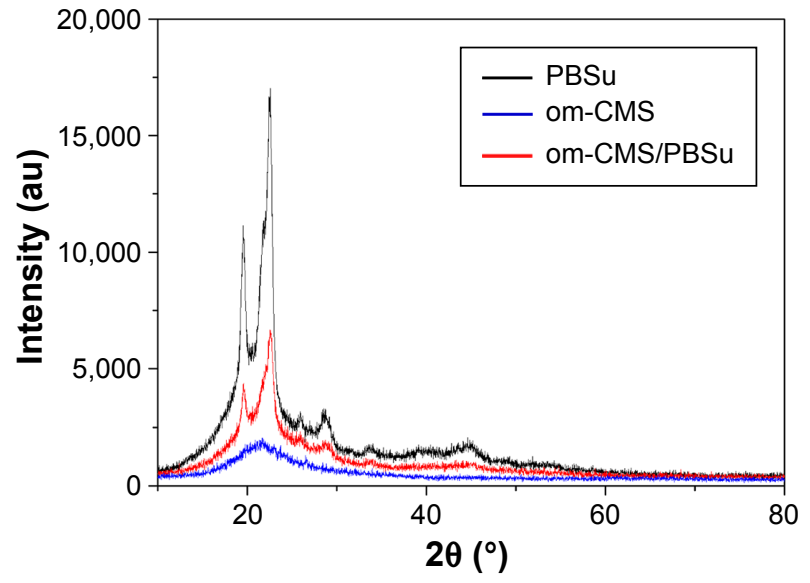

Figure 4 Wide-angle XRD patterns of PBSu, om-CMS and om-CMS/PBSu. Abbreviations: XRD, X-ray diffraction; PBSu, polybutylene succinate; om-CMS, ordered mesoporous calcium-magnesium silicate. degradation rate, $\mathrm{pH}$ variation, and changes in surface morphology.

Figure 5 shows the Scanning Electron Microscopy micrographs and photographs of PBSu and om-CMS/PBSu scaffolds before and after immersion in SBF for 5 days. The obvious difference in surface morphology was found before and after soaking the scaffold in SBF. The PBSu with a rough surface contained some columnar crystals (Figure 5A) before it was soaked in SBF. After soaking in SBF, it can be observed that the surface morphology of the PBSu scaffold has been degraded and the crystals were dissolved by the SBF (Figure 5C). The surface morphology of the om-CMS/ PBS scaffold was similar to PBSu but with less columnar crystals prior to soaking it in SBF (Figure 5B). This could be due to the blending of the amorphous om-CMS powder. After soaking in SBF, however, the surfaces of om-CMS/ $\mathrm{PBSu}$ were covered with a faveolate structure (Figure 5D); this was different than that observed in PBSu upon soaking in SBF. Notably, the degradation of om-CMS/PBSu was more severe than that of $\mathrm{PBSu}$ indicating that the degradation rate of the PBSu scaffold is increased by addition of om-CMS. These results suggested that the mesoporous structure of the om-CMS/PBSu scaffold enhances the biocompatibility as 

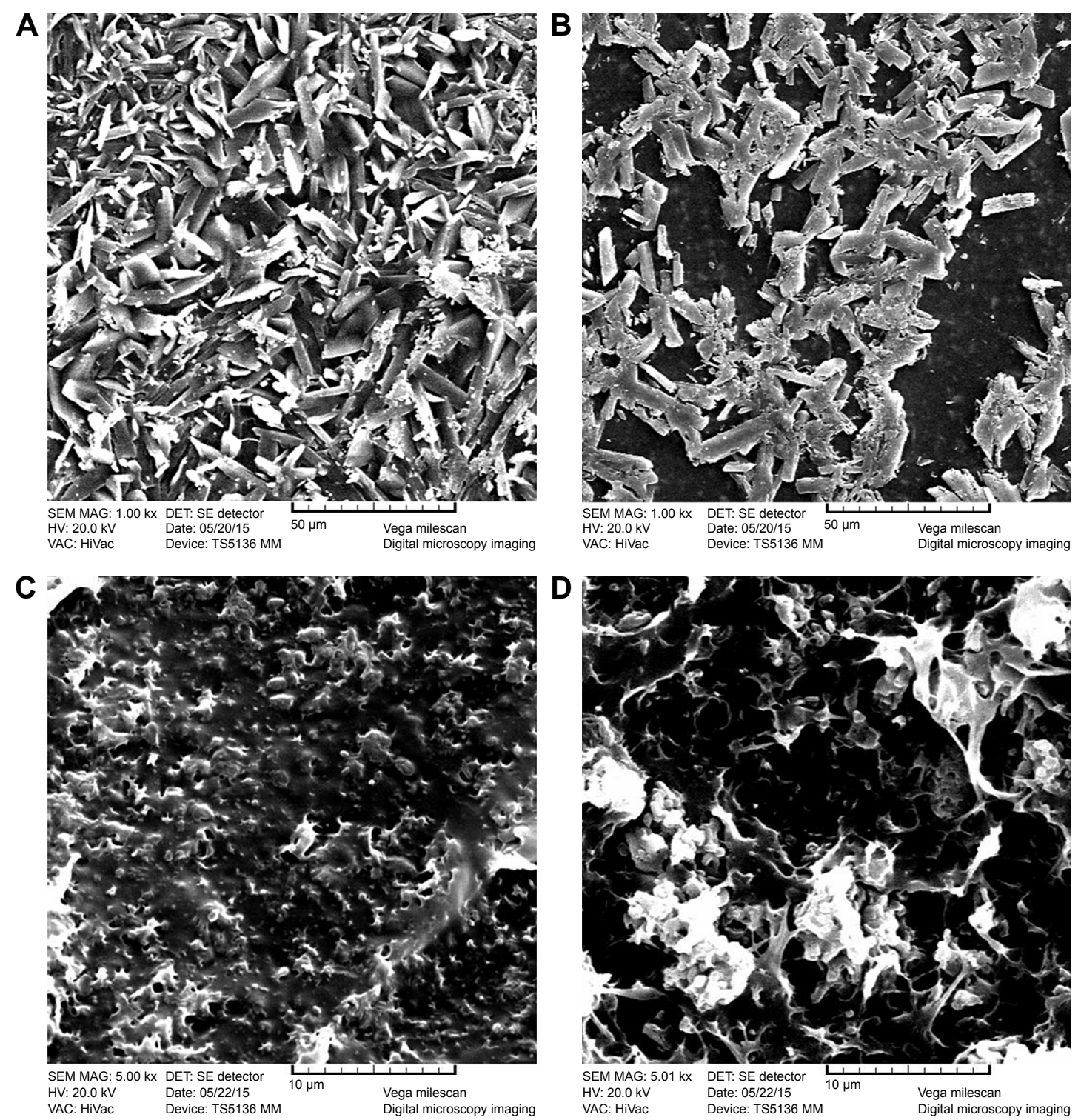

Figure 5 SEM micrographs of PBSu and om-CMS/PBSu before and after SBF immersion test.

Notes: SEM micrographs of PBSu (A), om-CMS/PBSu (B), and samples after 5 days immersion in the SBF. After soaking in SBF, the surface of PBSu scaffold (C) was degraded and om-CMS/PBSu (D) was observed to be covered with faveolate structure.

Abbreviations: PBSu, polybutylene succinate; om-CMS, ordered mesoporous calcium-magnesium silicate; SBF, simulated body fluid; SEM, scanning electron microscopy.

well as the chances of osseointegrated interface formation after implantation.

The changes in $\mathrm{pH}$ of the SBF solution after immersion of the PBSu and om-CMS/PBSu scaffolds for various durations are shown in Figure 6A. It was found that the $\mathrm{pH}$ of the PBSu solution decreased slowly from 7.4 to 7.1 during the first 8 weeks. A sharp decrease was noticed from week 9 onward and finally a $\mathrm{pH}$ of 5.6 was detected at the end of week 12 . However, the $\mathrm{pH}$ value of the om-CMS/PBSu containing SBF increased slowly in the first 2 weeks and then showed a slow decrease in the following 10 weeks. The $\mathrm{pH}$ of the SBF solution containing om-CMS/PBSu was 7.0 at week 12. Polyester is chemically degraded by hydrolysis and $\mathrm{pH}$ is one of the factors that influences the velocity of this hydrolysis through autocatalysis. ${ }^{24}$ The PBSu scaffold might be degrading by a random chain scission by ester hydrolysis in a process autocatalyzed by the generation of carboxylic acid end groups that accelerate degradation and $\mathrm{pH}$ decrease by acid catalysis after 8 weeks of immersion. The degradation of om-CMS in the om-CMS/PBSu scaffold is known to occur within the first few days of exposure to the physiological media. This is mainly due to the mesoporous structure providing a high contact interface for the SBF. The degradation of $\mathrm{Mg}$ and $\mathrm{Ca}$ in the om-CMS is described by the following reactions. ${ }^{25}$

$$
\mathrm{Mg} / \mathrm{Ca}+2 \mathrm{H}_{2} \mathrm{O} \rightarrow \mathrm{Mg}(\mathrm{OH})_{2} / \mathrm{Ca}(\mathrm{OH})_{2}+\mathrm{H}_{2}
$$



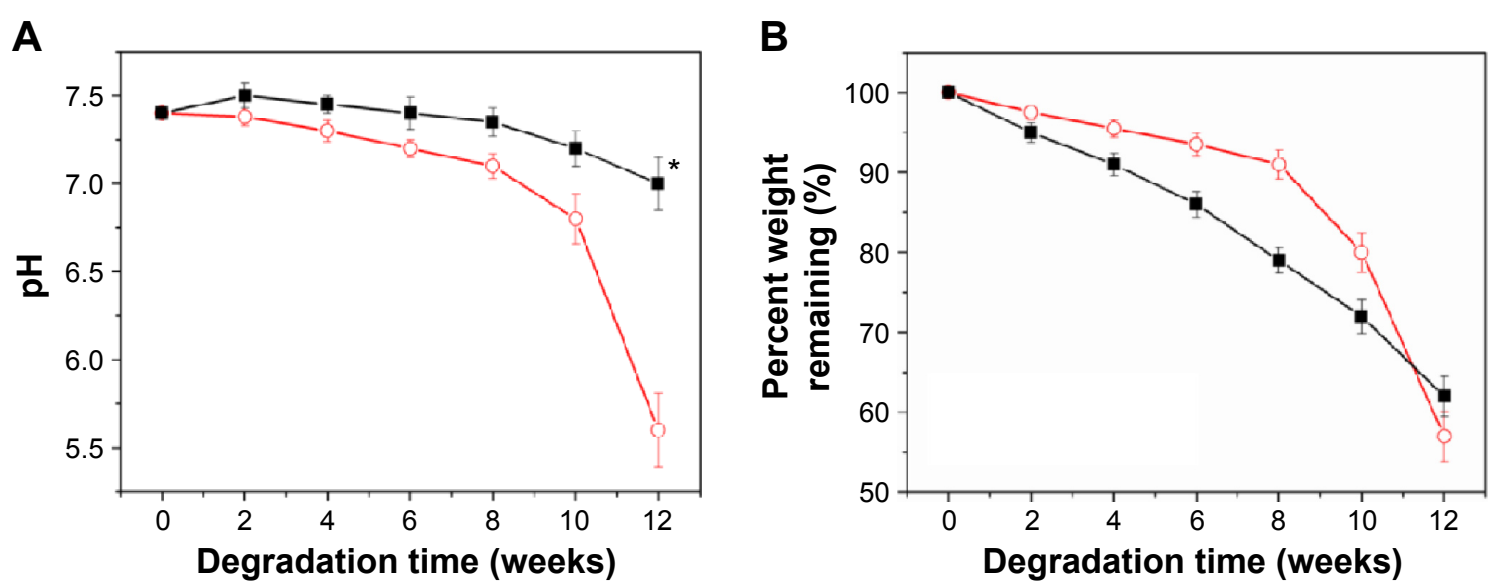

$$
\text { - - PBSu - - om-CMS/PBSu }
$$

Figure $6 \mathrm{pH}$ and weight changes of PBSu and om-CMS/PBSu scaffolds in SBF immersion test.

Notes: (A) Variation of $\mathrm{pH}$ of the SBF solution with degradation time for PBSu and om-CMS/PBSu scaffolds. $* \mathrm{P}<0.05$ compared to PBSu scaffold. (B) Percent of weight remaining compared with day 0 values as a function of degradation time for PBSu and om-CMS/PBSu scaffolds.

Abbreviations: SBF, simulated body fluid; PBSu, polybutylene succinate; om-CMS, ordered mesoporous calcium-magnesium silicate.

$$
\mathrm{Mg}(\mathrm{OH})_{2} / \mathrm{Ca}(\mathrm{OH})_{2}+2 \mathrm{Cl}^{-} \rightarrow \mathrm{MgCl}_{2} / \mathrm{CaCl}_{2}+2 \mathrm{OH}^{-}
$$

Degradation of om-CMS is accompanied by an alkalization of the corrosive media due to the production of hydroxide ions $\left(\mathrm{OH}^{-}\right)$. A pH increase during the first few days of immersion has been reported by several in vitro studies. ${ }^{26,27}$ The alkalization of the om-CMS and the acidification of the PBSu solutions reach equilibrium after 2 weeks while the $\mathrm{pH}$ of the SBF solution containing om-CMS/PBSu stabilizes. This finding demonstrates that the om-CMS/PBSu scaffold is a superior biological material than the other two scaffolds. This stable neutral $\mathrm{pH}$ may promote cell proliferation and high biological safety. ${ }^{28}$

Figure 6B represents the percentage of weight remaining of the om-CMS/PBSu and PBSu scaffolds compared with day 0 values as a function of the incubation time. The weight of PBSu was $91.3 \% \pm 2.1 \%$ of the initial weight after 8 weeks of degradation in SBF solution and then decreased dramatically to $57.1 \% \pm 3.2 \%$ at the end of 12 weeks. The om-CMS/PBSu scaffold showed steady degradation over time and the weight loss rate was approximately $3 \%$ per week. Biomaterials used for bone regeneration should be degradable and gradually replaced by new bone when implanted in vivo. The constant degradation rate of the om-CMS/PBSu scaffold can be attributed to its mesoporous structure and large surface area and this could improve the osteocompatibility in vivo, ultimately benefiting the physicochemical interaction between the cells and the material. ${ }^{29}$

\section{Bioactivity of om-CMS/PBSu in vitro}

The X-ray diffraction patterns of om-CMS/PBSu after soaking into $\mathrm{SBF}$ for 5 days are shown in Figure 7A.
A new crystal peak was observed between $10^{\circ}$ and $20^{\circ}$ after immersion. This could be the signal of phosphorus indicating apatite formation on the om-CMS/PBSu surface. The inductively coupled plasma results (Figure 7B) show changes in $\mathrm{Mg}$ and $\mathrm{Ca}$ ion concentration of the solution after immersing om-CMS/PBSu into SBF for different time durations. The $\mathrm{Mg}$ and $\mathrm{Ca}$ ion concentrations of om-CMS/ $\mathrm{PBSu}$ in SBF increased at different rates with soaking time and reached 47 and $261 \mathrm{mg} / \mathrm{L}$ at 96 hours, respectively. It has been reported that ion-dissolution products containing $\mathrm{Ca}, \mathrm{Si}$, and $\mathrm{Mg}$ from bioactive glasses and ceramics could stimulate the nucleation of bioactive minerals. ${ }^{30}$ In addition, SBF is a super saturated calcium phosphate solution and its chemical stimulus may activate the nucleation of bioactive minerals including phosphate and carbonate groups and the induction of bioactivity can be carried out by negatively charged groups. In particular, the formation of silanol (-Si-OH) on the surface of a scaffold is known to be beneficial for nucleation of bioactive products. The positively charged $\mathrm{Ca}^{2+}$ and $\mathrm{Mg}^{2+}$ attract negatively charged groups $\mathrm{PO}_{4}{ }^{3-}$ and $\mathrm{CO}_{3}{ }^{2-}$ in the SBF and eventually lead to the formation of a phosphate layer on the surface of the scaffold. ${ }^{31}$ This may in turn be beneficial in enhancing the chances of formation of an osseointegrated interface and cell proliferation after implantation.

\section{Cell adhesion}

The effect of adsorbed FN on adhesion of MC3T3-E1 cells to the om-CMS/PBSu and PBSu substrates after 6 hours of seeding is shown in Figure 8. The cells reach an equilibrium adherence level after 6 hours and then spread on 
A

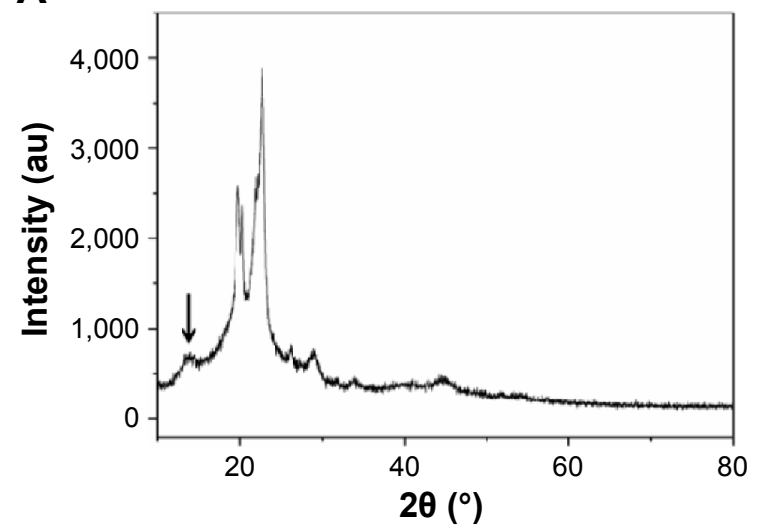

B

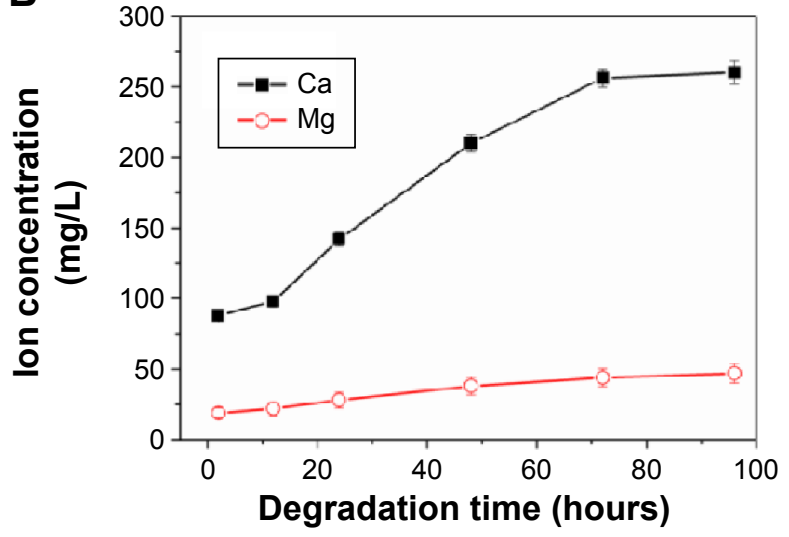

Figure 7 XRD pattern and released ion concentration of om-CMS/PBSu after immersion in the SBF.

Notes: (A) XRD patterns of om-CMS/PBSu after soaking in SBF for 5 days. Arrow represents the apatite peak. (B) Concentration of released Mg ${ }^{2+}$ and Ca ${ }^{2+}$ for om-CMS/ PBSu scaffold.

Abbreviations: XRD, X-ray diffraction; om-CMS, ordered mesoporous calcium-magnesium silicate; PBSu, polybutylene succinate; SBF, simulated body fluid.
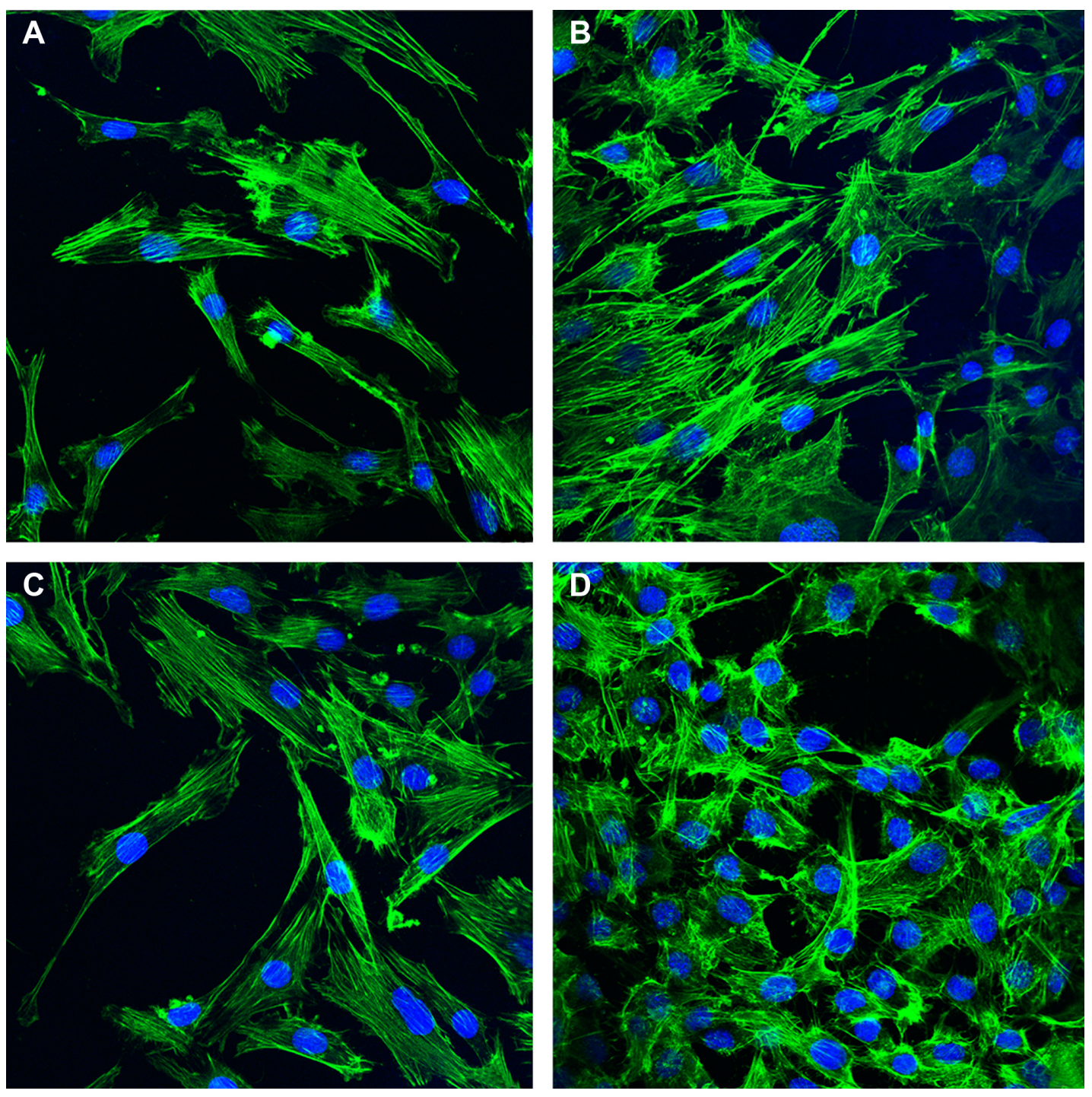

Figure 8 Fluorescence microscopy images of MC3T3-EI cells on PBSu substrates without and with FN treatment ([A] and [C], respectively) and om-CMS/PBSu substrates without and with FN treatment ([B] and [D], respectively). Cells were stained with Acti-stain 488 phalloidin (green) and DAPI (blue).

Abbreviations: PBSu, polybutylene succinate; FN, fibronectin; om-CMS, ordered mesoporous calcium-magnesium silicate. 
the surface. Figure 8C and D clearly shows that the presence of FN increases cell attachment to either substrate. More MC3T3-E1 cells were observed to attach to the om-CMS/ PBSu composite than to the PBSu indicating that the use of om-CMS/PBSu could promote the attachment of MC3T3-E1 cells. It has been shown that materials with a mesoporous structure promote protein unfolding and adsorbed protein density. ${ }^{32}$ Thus, the om-CMS/PBSu substrate could promote increased FN binding by providing a larger surface area and pore volume thereby enhancing cell adhesion significantly. In addition, the hydrophilic surface of the om-CMS/PBSu is beneficial for cell attachment, spreading, and cytoskeletal organization. ${ }^{33}$ The silanol groups generated by om-CMS can bind to various functional groups of proteins via hydrogen bonds or long-range electrostatic ionic amine bonds ( $\mathrm{Si}^{-} \mathrm{O}^{-}$ ${ }^{+} \mathrm{H}_{3} \mathrm{~N}$ ), thus producing a favorable surface environment for cell attachment. ${ }^{34}$ Further, $\mathrm{Ca}^{2+}$ and $\mathrm{Mg}^{2+}$ ions on the surface of the om-CMS/PBSu act as ligands for osteoblast favored protein adsorption due to positive electrostatic attraction. ${ }^{35}$ Therefore, the om-CMS/PBSu composite could exert a positive effect on cell attachment and spreading.

\section{Cell proliferation}

The proliferation of MC3T3-E1 cells cultured on the om$\mathrm{CMS} / \mathrm{PBSu}$ and PBSu surfaces was analyzed using a Cell Counting Kit-8 assay as shown in Figure 9. Cells grown on the composites displayed a steady continuous increase in proliferation from day 1 to day 7, suggesting no negative effects on cell growth. Cells grown on PBSu exhibited no significant increase in proliferation from day 4 to day 7 . The relative proliferation rate of cells grown on the om-CMS/ $\mathrm{PBSu}$ was significantly higher than those grown on $\mathrm{PBSu}$,

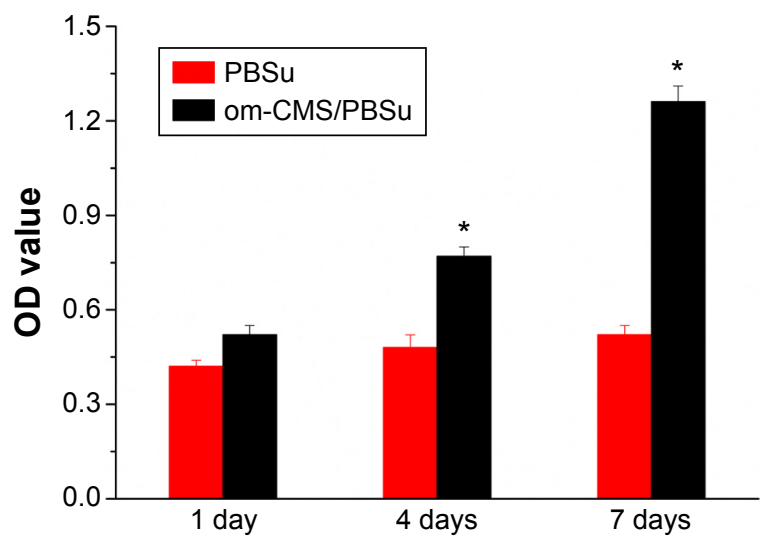

Figure 9 MC3T3-EI cell proliferation on PBSu and om-CMS/PBSu substrates for I, 4, and 7 days.

Notes: The om-CMS/PBSu showed approximately 2.4 times higher cell proliferation after 7 days of culture compared with PBSu; $* P<0.05$ compared to PBSu.

Abbreviations: PBSu, polybutylene succinate; om-CMS, ordered mesoporous calcium-magnesium silicate; OD, optical density. indicating that om-CMS/PBSu promotes the proliferation of MC3T3-E1 cells. Studies have shown that the release of appropriate quantities of $\mathrm{Si}$ ions from silicate-containing materials plays an important role in stimulating the proliferation of osteoblasts and bone marrow stromal cells. ${ }^{36}$ Further, appropriate levels of $\mathrm{Ca}^{2+}$ and $\mathrm{Mg}^{2+}$ are known to stimulate osteoblast proliferation by modulating the expression of specific ion channel isoforms in osteoblasts. In addition, the formation of an apatite layer on the composite surface is known to be beneficial for osteoblast proliferation.

\section{Conclusion}

In this study, a bioactive scaffold was fabricated by incorporating om-CMS into $\mathrm{PBSu}$ using a process of compounding and molding. The resulting om-CMS/PBSu scaffold had a large surface area of $891 \mathrm{~m}^{2} / \mathrm{g}$ and highly ordered channels of pores of approximately $13 \mathrm{~nm}$ in size. The om-CMS/ PBSu is degradable with a weight loss ratio of $38 \%$ after immersion in SBF solution for 12 weeks with a relatively constant $\mathrm{pH}$ value of the solution. The om-CMS/PBSu showed good bioactivity in vitro, which could induce apatite formation on its surfaces after immersion in SBF. Further, the use of om-CMS/PBSu significantly increased attachment, proliferation, and spreading of MC3T3-E1 cells compared to PBSu. The positive effects of om-CMS/PBSu on cellular responses are most likely due to the mesoporous structure of this surface, formation of apatite-layer, and the $\mathrm{Si}, \mathrm{Ca}$, and $\mathrm{Mg}$ ions released from the composite. Due to its increased bioactivity and biocompatibility the om-CMS/PBSu scaffold is believed to be a promising orthopedic implant material for application in bone repair.

\section{Acknowledgments}

The authors gratefully acknowledge the financial support from the National Nature Science Foundation of China (grant number: 81201177 and 81571796), Youth Innovation Promotion Association, CAS, and the SA-SIBS Scholarship Program. The authors would like to thank Dr S Liu and Dr Shangchun Guo for their excellent technical assistance.

\section{Disclosure}

The authors report no conflicts of interest in this work.

\section{References}

1. Ingavle GC, Leach JK. Advancements in electrospinning of polymeric nanofibrous scaffolds for tissue engineering. Tissue Eng Part B Rev. 2014; 20(4):277-293.

2. Murphy WL, Peters MC, Kohn DH, Mooney DJ. Sustained release of vascular endothelial growth factor from mineralized poly(lactide-coglycolide) scaffolds for tissue engineering. Biomaterials. 2000;21(24): 2521-2527. 
3. Serra IR, Fradique R, Vallejo MCS, Correia TR, Miguel SP, Correia IJ. Production and characterization of chitosan/gelatin/ $\beta$-TCP scaffolds for improved bone tissue regeneration. Mater Sci Eng C Mater Biol Appl. 2015;55:592-604.

4. Lv YM, Yu QS. Repair of articular osteochondral defects of the knee joint using a composite lamellar scaffold. Bone Joint Res. 2015;4(4): $56-64$.

5. Zhou P, Xia Y, Cheng X, Wang P, Xie Y, Xu S. Enhanced bone tissue regeneration by antibacterial and osteoinductive silica-HACC-zein composite scaffolds loaded with rhBMP-2. Biomaterials. 2014;35(38): 10033-10045.

6. Lu W, Ji K, Kirkham J, et al. Bone tissue engineering by using a combination of polymer/Bioglass composites with human adipose-derived stem cells. Cell Tissue Res. 2014;356(1):97-107.

7. Van Rie J, Declercq H, Van Hoorick J, et al. Cryogel-PCL combination scaffolds for bone tissue repair. J Mater Sci Mater Med. 2015;26(3):123.

8. Stoyanova N, Paneva D, Mincheva R, et al. Poly(L-lactide) and poly(butylene succinate) immiscible blends: from electrospinning to biologically active materials. Mater Sci Eng CMater Biol Appl. 2014;41: 119-126.

9. Wang H, Xu M, Wu Z, Zhang W, Ji J, Chu PK. Biodegradable poly(butylene succinate) modified by gas plasmas and their in vitro functions as bone implants. ACS Appl Mater Interfaces. 2012;4(8): 4380-4386.

10. Félix Lanao RP, Sariibrahimoglu K, Wang H, Wolke JG, Jansen JA, Leeuwenburgh SC. Accelerated calcium phosphate cement degradation due to incorporation of glucono-delta-lactone microparticles. Tissue Eng Part A. 2014;20(1-2):378-388.

11. Arphavasin S, Singhatanadgit W, Ngamviriyavong P, Janvikul W, Meesap P, Patntirapong S. Enhanced osteogenic activity of a poly (butylene succinate)/calcium phosphate composite by simple alkaline hydrolysis. Biomed Mater. 2013;8(5):055008.

12. Wang H, Ji J, Zhang W, et al. Biocompatibility and bioactivity of plasma-treated biodegradable poly(butylene succinate). Acta Biomater. 2009;5(1):279-287.

13. Abdollahi S, Ma AC, Cerruti M. Surface transformations of Bioglass 45S5 during scaffold synthesis for bone tissue engineering. Langmuir. 2013;29(5):1466-1474.

14. Shah Mohammadi M, Chicatun F, Stähli C, Muja N, Bureau MN, Nazhat SN. Osteoblastic differentiation under controlled bioactive ion release by silica and titania doped sodium-free calcium phosphate-based glass. Colloids Surf B Biointerfaces. 2014;121:82-91.

15. Fernández JM, Molinuevo MS, McCarthy AD, Cortizo AM. Strontium ranelate stimulates the activity of bone-specific alkaline phosphatase: interaction with $\mathrm{Zn}(2+)$ and $\mathrm{Mg}(2+)$. Biometals. 2014;27(3): 601-607.

16. Singh LP, Bhattacharyya SK, Kumar R, et al. Sol-Gel processing of silica nanoparticles and their applications. Adv Colloid Interface Sci. 2014;214C:17-37.

17. Chen $B$, Wang $Z$, Quan $G$, et al. In vitro and in vivo evaluation of ordered mesoporous silica as a novel adsorbent in liquisolid formulation. Int $J$ Nanomedicine. 2012;7:199-209.

18. Wanyika H. Controlled release of agrochemicals intercalated into montmorillonite interlayer space. ScientificWorldJournal. 2014;2014: 656287.

International Journal of Nanomedicine

\section{Publish your work in this journal}

The International Journal of Nanomedicine is an international, peerreviewed journal focusing on the application of nanotechnology in diagnostics, therapeutics, and drug delivery systems throughout the biomedical field. This journal is indexed on PubMed Central, MedLine, CAS, SciSearch ${ }^{\circledR}$, Current Contents ${ }^{\circledR} /$ Clinical Medicine,
19. Kokubo T, Takadama H. How useful is SBF in predicting in vivo bone bioactivity? Biomaterials. 2006;27(15):2907-2915.

20. Lee HN, Kim CH, Song GG, Cho SW. Effects of IL-3 and SCF on histamine production kinetics and cell phenotype in rat bone marrowderived mast cells. Immune Netw. 2010;10(1):15-25.

21. Zheng BJ, Qi B, Hu YL, Zhang F, Liu HF. Electro-catalytic activity of palladium modified nitrogen doped titania nanoparticles. $J$ Nanosci Nanotechnol. 2014;14(5):3527-3531.

22. Zhang JH, Zhao SC, Zhu YF, et al. Three-dimensional printing of strontium-containing mesoporous bioactive glass scaffolds for bone regeneration. Acta Biomater. 2014;10(5):2269-2281.

23. Zhu YF, Wu CT, Ramaswamy Y, et al. Preparation, characterization and in vitro bioactivity of mesoporous bioactive glasses (MBGs) scaffolds for bone tissue engineering. Micropor Mesopor Mater. 2008;112(1-3): 494-503.

24. Wang H, Ji J, Zhang W, et al. Biocompatibility and bioactivity of plasma-treated biodegradable poly(butylene succinate). Acta Biomater. 2009;5(1):279-287.

25. Yazdimamaghani M, Razavi M, Vashaee D, Tayebi L. Surface modification of biodegradable porous $\mathrm{Mg}$ bone scaffold using polycaprolactone/ bioactive glass composite. Mater Sci Eng C Mater Biol Appl. 2015; 49(4):436-444.

26. Wong HM, Yeung KW, Lam KO, et al. A biodegradable polymer-based coating to control the performance of magnesium alloy orthopaedic implants. Biomaterials. 2010;31(8):2084-2096.

27. Yazdimamaghani M, Razavi M, Vashaee D, Tayebi L. Development and degradation behavior of magnesium scaffolds coated with polycaprolactone for bone tissue engineering. Mater Lett. 2014;132:106-110.

28. Maỉborodin IV, Kuznetsova IV, Shevela AI, Barannik MI, Manaev AA, Maiborodina VA. Tissue reactions to the use of implants manufactured from lactic acid polymers. Morfologiia. 2014;146(4):78-89.

29. Baino F, Fiorilli S, Mortera R, et al. Mesoporous bioactive glass as a multifunctional system for bone regeneration and controlled drug release. J Appl Biomater Funct Mater. 2012;10(1):12-21.

30. Pan Y, Chen C, Wang D, Huang D. Dissolution and precipitation behaviors of silicon-containing ceramic coating on $\mathrm{Mg}-\mathrm{Zn}-\mathrm{Ca}$ alloy in simulated body fluid. Colloids Surf B Biointerfaces. 2014;122:746-751.

31. Jones JR. Review of bioactive glass: from Hench to hybrids. Acta Biomater. 2013;9(1):4457-4486.

32. Lee CH, Lang J, Yen CW, Shih PC, Lin TS, Mou CY. Enhancing stability and oxidation activity of cytochrome $\mathrm{C}$ by immobilization in the nanochannels of mesoporous aluminosilicates. $J$ Phys Chem B. 2005;109(25):12277-12286.

33. Allen LT, Tosetto M, Miller IS, et al. Surface-induced changes in protein adsorption and implications for cellular phenotypic responses to surface interaction. Biomaterials. 2006;27(16):3096-3108.

34. Wu CT, Chang J, Ni SY, Wang JY. In vitro bioactivity of akermanite ceramics, J Biomed Mater Res A. 2006;76(1):73-80.

35. Walmsley GG, McArdle A, Tevlin R, et al. Nanotechnology in bone tissue engineering. Nanomedicine. 2015;11(5):1253-1263.

36. Shi M, Zhou Y, Shao J, et al. Stimulation of osteogenesis and angiogenesis of hBMSCs by delivering $\mathrm{Si}$ ions and functional drug from mesoporous silica nanospheres. Acta Biomater. 2015;21:178-189.

Journal Citation Reports/Science Edition, EMBase, Scopus and the Elsevier Bibliographic databases. The manuscript management system is completely online and includes a very quick and fair peer-review system, which is all easy to use. Visit http://www.dovepress.com/ testimonials.php to read real quotes from published authors. 